furthermore let

$$
\left|f_{i}(x, t, y)\right| \leqq M \quad(i=1,2) .
$$

Then if $\rho$ satisfies the conditions

$$
\rho \leqq \alpha, \quad \rho \leqq \frac{\beta}{M}, \quad \rho \leqq \frac{\gamma}{2 A M},
$$

there exists, in the interval $\left|x-x_{0}\right| \leqq \rho$, one and only one continuous solution $y(x)$ of the integral equation (1).

The proof is entirely similar to that of Theorem I.

Cornelu University,

January 10, 1913.

\title{
A THEOREM ON ASYMPTOTIC SERIES.
}

BY MR. VINCENT C. POOR.

Theorem: If $f(z)$ is not holomorphic at $z=0$, but is formally developable into a Maclaurin series, and if $w$ is asymptotic to $a_{1} / z+a_{2} / z^{2}+\cdots$ (written: $\left.w \sim a_{1} / z+a_{2} / z^{2}+\cdots\right)$, then $f(w)$ has an asymptotic representation.*

To prove this theorem take $f(z)$ in the form

$$
f(z)=f(0)+f^{\prime}(0) z+\frac{f^{\prime \prime}(0)}{2 !} z^{2}+\cdots
$$

Since

$$
+\frac{f^{(n)}(0) z^{n}}{n !}+\int_{0}^{z} f^{(n+1)}(t) \cdot \frac{(z-t)^{n}}{n !} d t .
$$

$$
w \sim \frac{a_{1}}{z}+\frac{a_{2}}{z^{2}}+\cdots
$$

$w$ may be written

$$
w=\frac{a_{1}}{z}+\frac{a_{2}}{z^{2}}+\cdots+\frac{a_{n}+\epsilon_{1 n z}}{z^{n}},
$$

where, according to the Poincaré definition $\dagger$ for an asymptotic

* This theorem is the "résultat préalablement obtenu" referred to in Professor Ford's paper in the Bulletin of the French Society for 1911. See Bulletin Société math. de France, vol. 40 (1912), fascicule 1 under "Erratum du Tome XXXIX."

† Poincaré, Acta Mathematica, vol. 8 (1886), p. 296. 
series,

$$
\lim _{z \doteq \infty} \epsilon_{1 n z}=0 .
$$

Replacing $z$ by $w$ in (1) and collecting coefficients of like powers, the following equation results:

$$
f(w)=f(0)+\frac{A_{1}}{z}+\frac{A_{2}}{z^{2}}+\cdots+\frac{A_{n}+\epsilon_{z}}{z^{n}}+R,
$$

where

$$
R=\int_{0}^{w} f^{(n+1)}(t) \cdot \frac{(w-t)^{n}}{n !} d t
$$

and where the $A_{i}(i=1, \cdots, n)$ are readily determined from the substitution.

and

$$
\epsilon_{z}=\epsilon_{1 n z}+\epsilon_{2 n z}+\cdots+\epsilon_{n n z}
$$

$$
\epsilon_{\text {in } z} / z^{n} \quad(i=1, \cdots, n)
$$

are the remainders after $n$ terms of $w, w^{2}, w^{3}, \cdots, w^{n}$, respectively. Since

$$
\lim _{z \doteq \infty} \epsilon_{\text {in } z}=0 \quad(i=1, \cdots, n),
$$

it follows that

$$
\lim _{z \doteq \infty} \epsilon_{z}=0
$$

To satisfy the Poincaré definition of an asymptotic series, it remains to show that

$$
\lim _{z \doteq \infty} z^{n} R=0 .
$$

For $z$ real this last condition may be shown to hold as follows: $(w-t)^{n}$ does not change sign in the interval $0 \leqq t \leqq w$, therefore the first law of the mean for integrals may be applied. Making this application, it thus obtains that

(4) $z^{n} R \equiv \frac{z^{n}}{n !} \int_{0}^{w} f^{(n+1)}(t) \cdot(w-t)^{n} d t=\frac{z^{n} \theta_{z} M}{n !} \int_{0}^{w}(w-t)^{n} d t$,

where $M$ is the maximum value of $\left|f^{(n+1)}(t)\right|$ in the interval $0 \leqq t \leqq w$, and where $-1 \leqq \theta_{z} \leqq 1$. Evaluating the integral 
in (4) and taking the limit, it is found that

$$
\lim _{z \doteq \infty} z^{n} R=\lim _{z=\infty} \frac{z^{n} \cdot \theta_{z} M \cdot w^{n+1}}{(n+1) !}=0 .
$$

Hence by definition

$$
f(w) \sim f(0)+\frac{A_{1}}{z}+\frac{A_{2}}{z^{2}}+\cdots .
$$

The form I have selected for the remainder in (1) readily applies for $z$ complex. Taking the path of integration as a straight line from $z=0$ to $z=w$ and writing $t=r e^{i \theta}, w=w_{0} e^{i \theta}$, then

$$
\left|z^{n}\right| \cdot|R| \cdot \leqq \frac{\left|z^{n}\right| \cdot M}{n !} \int_{0}^{w_{0}}\left|w_{0}-r\right|^{n} \cdot d r,
$$

$M$ being the maximum value of $\left|f^{(n+1)}(t)\right|$ in the interval of integration. But

$$
\lim _{z \doteq \infty}\left|z^{n}\right| \cdot|R| \leqq \lim _{z \doteq \infty} \frac{\left|z^{n}\right| M \cdot w_{0}^{n}}{n !} \int_{0}^{w_{0}} d r=\lim _{z \doteq \infty} \frac{z^{n} M \cdot w_{0}{ }^{n+1}}{n !}=0 .
$$

Thus (5) holds for $z$ complex.

That the divergent series

$$
f(0)+f^{\prime}(0) z+\frac{f^{\prime \prime}(0)}{2 !} z^{2}+\cdots
$$

is asymptotic at $z=0$, or replacing $z$ by $1 / z$ in (6), at $z=\infty$, is evident since $w$ may be chosen as $1 / z$ in (5). (6) is therefore an asymptotic representation for $f(z)$ in the vicinity of $z=0$, or if $w$ be taken as $1 / z$ in (5),

$$
f\left(\frac{1}{z}\right) \sim f(0)+\frac{f^{\prime}(0)}{z}+\frac{f^{\prime \prime}(0)}{2 ! z^{2}}+\cdots .
$$

AnN Arbor, Mich.,

January, 1913. 